American J. of Engineering and Applied Sciences 2 (1): 70-75, 2009

ISSN 1941-7020

(C) 2009 Science Publications

\title{
Numerical and Analytical Study of Exhaust Gases Flow in Porous Media with Applications to Diesel Particulate Filters
}

\author{
Suleiman Abu-Ein \\ Department of Engineering Technology, Al-Balqua Applied University, Amman, Jordan \\ Mechanical Engineering, Al-Balqua Applied University Amman 11134 Jordan
}

\begin{abstract}
Problem statement: The importance of diesel particulate filter has been increased later because of its ability in making emission reduction of the exhaust gasses. Diesel Particulate Filters (DPF) do two important jobs: gas emissions reduction and noise reduction, the pores spread inside the DPF store some gases and soot emissions and on the other side it prevents some sound or noise to be gone outside, so it works as a noise reduction device or unit. Diesel particulate filter is considered as a porous media, it's very important to study the flow behavior in such media in order to redesign dimensions, specifications and location of diesel particulate filter on the exhaust pipes. Approach: The flow of gasses through the Diesel Particulate Filters (DPF) unit is studied here analytically and numerically to reach for the best equation describes the real nature of flow behavior. The analytical model is built to find the velocity of flow, pressure drop and nature of flow-laminar or turbulent- at two conditions at cold conditions $\left(25^{\circ} \mathrm{C}\right)$ and hot conditions $\left(500^{\circ} \mathrm{C}\right)$. Also the problem is solved numerically using some appropriate software. A comparison was made between both analytical and numerical results especially the pressure drop effect. Some appropriate software used to describe the reality of the gasses flow through porous media. Results: It was found that the nature gases flow in DPF is laminar; a pressure drop through the DPF was occurred, gases axial velocity, vertical velocity, temperature are all decrease as such gases go through the filter. Vertical velocity's values were very small compared with the axial velocity's values. Conclusion/Recommendations: Gases pressure is decreased as such gases flow along the DPF unit and exhaust system, so there is a pressure drop. Temperature distribution and profiles showed that the values of temperature have a fluctuation along the DPF unit. Axial and vertical velocities were decreasing as the gases flow through the DPF unit and the exhaust system. It is recommended to study the flow as a non-linear 3D problem.
\end{abstract}

Key words: Porous media, laminar gases flow, darcy's equation, reynolds number and temperature and pressure distribution

\section{INTRODUCTION}

The flow in the pure fluid region is usually described by the Navier-Stokes system of equations. The most popular models for the flow in the porous media are those suggested by Darcy and by Brinkman, the flow of several components and several phases through a porous medium is generally described by introducing macroscopic mass-balance equations under the form of generalized dispersion equations. Porous medium consists of a matrix with a large amount of microscopic pores and throats which are typically narrow tubes where fluid can pass through. Soil and sand are both examples of porous media and fluid flow in a porous medium can be considered as pouring a cup of water over soil and letting the water flow into the soil due to the gravitational forces. The description of flow in porous media is extremely difficult because of the complexity of the medium. Even though flow in a single tube is given by simple equations, the network of the tubes is impossible to know in detail. Fluid flow through a porous medium is often given by the phenomenological Darcy equation. Consider a porous medium of absolute permeability $\sigma$ in a homogeneous gravitation field where one fluid of viscosity $\mu$ is injected though the medium by applying a pressure gradient $\Delta \mathrm{P}$ across the matrix. Then the flow rate $\mathrm{Q}$ of the fluid through the medium is given by Darcy's equation, such that:

$\mathrm{Q}=-\left(\frac{\sigma \mathrm{A}}{\mu}\right)\left(\frac{\Delta \mathrm{P}}{\mathrm{L}}\right)$

This is a linear law, similar to Newton's law of viscosity, Ohm's law of electricity. In Eq. 1, Q is the volumetric flow rate (or "discharge"), A the normal cross-sectional area of the sample, $\mathrm{L}$ the length of the sample in the macroscopic flow direction, $\mathrm{P}$ piezometric pressure.

There are four conditions that are required for this equ to be valid: 
- Creeping flow-The modified Reynold's number based on superficial velocity must be on the order of 1

- The porous media itself is not reactive with the flowing fluid

- No accumulation

- Single-phase flow

Using Eq. 1, it is possible to determine the permeability $(\sigma)$ of the porous media. Darcy's equation is applied for those porous media which have regions with a smaller pores which also treated as a permeable medium as in the case presented (partially porous media not highly porous). The most general equation describes the matching of boundary conditions at an interface between the larger pores and the permeable medium is the Brinkman's equation, such that:

$$
\Delta \mathrm{P}=-\frac{\mu}{\sigma} \mathrm{V}+\mu_{\mathrm{e}} \nabla^{2} \mathrm{~V}
$$

Where:

$\mathrm{V}=$ Fluid velocity

$\mu=$ Fluid viscosity,

$\mu_{\mathrm{e}}=$ Effective fluid viscosity,

$\sigma=$ Permeability,

$\Delta \mathrm{P}=$ Pressure drop

The equation satisfied two boundary conditions at the pore/permeable medium interface which are continuity of the fluid velocity and the shear stress ${ }^{[1,5,7]}$.

Although liquids display a permeability that depends on the media only, gases display a permeability that also depends on the identity of the gas and the pressure differential across the media. This variation with the identity and pressure of the gas is known as slippage. This effect was originally detected with gas flow through capillary tubes and becomes more pronounced when the diameter of the capillary tubes approach the mean free path of the gas molecule. The mean free path is a function of the molecular weight and kinetic energy of a gas.

Flow in porous media requires a description of both the media and the flow. In the broadest sense an open pipe is a limiting case of a porous medium. Usually the number of holes or pores is sufficiently large that a volume average is needed to calculate pertinent proprieties. Pores which occupy some definite fraction of the bulk volume form a complex network of voids.

Flow in a porous medium is usually a creeping flow where the modified Reynolds number defined for a porous medium is less than one. In order to define the
Reynolds number for flow in porous media, the concept of hydraulic radius, $R_{H}$ can be used. The hydraulic radius is defined as the void volume of a porous medium divided by the surface area of the medium such that

$\mathrm{R}_{\mathrm{H}}=\frac{\text { void volume of porous medium }}{\text { surface area of porous medium }}$

If $\mathrm{V}_{\mathrm{p}}$ : is the particle volume and the surface area of the particle, $S_{P}$, for spherical particles, the specific surface, $\mathrm{B}$ (which has dimension $\mathrm{m}^{-1}$ ) can be defined as:

$$
\mathrm{B}=\frac{6}{\mathrm{D}}=\frac{\mathrm{S}_{\mathrm{P}}}{\mathrm{V}_{\mathrm{P}}}
$$

where $\mathrm{D}$ is the diameter of a particle.

The hydraulic radius can be rewritten in terms of the porosity, $\phi$, the number of particles, $\mathrm{N}$, the volume of the particles, $V_{P}$ and the surface area of a particle, $S_{P}$, in the following way:

$$
\mathrm{R}_{\mathrm{H}}=\frac{\phi \mathrm{V}_{\mathrm{P}}(\mathrm{N} / 1-\phi)}{\mathrm{S}_{\mathrm{P}} \mathrm{N}}=\frac{\phi \mathrm{V}_{\mathrm{P}}}{\mathrm{S}_{\mathrm{P}}(1-\phi)}
$$

For nonspherical particles, an effective particle diameter. $\mathrm{D}_{\mathrm{P}}$, can be defined as:

$$
\mathrm{D}_{\mathrm{P}}=\frac{6}{\mathrm{~B}}
$$

The discharge velocity, $\overline{\mathrm{u}}$ and the pore velocity, $\mathrm{v}$, are related by the mass balance as in the following equation:

$\overline{\mathrm{u}} \mathrm{A}_{\text {tot }}=\mathrm{vA}_{\text {void }}$

This gives the result:

$$
\overline{\mathrm{u}}=\phi_{\mathrm{A}} \mathrm{v}
$$

Hence Reynolds number for flow in a pipe is:

$$
\begin{aligned}
\operatorname{Re} & =\frac{\rho \overline{\mathrm{u}}^{2}}{\mu\left(\frac{\overline{\mathrm{u}} / \mathrm{D}}{\mathrm{D}}\right)} \\
& =\frac{\overline{\mathrm{u} D} \rho}{\mu}
\end{aligned}
$$

Where:

$\mu=$ Viscosity 
Am. J. Engg. \& Applied Sci., 2 (1): 70-75, 2009

$\overline{\mathrm{u}}=$ Discharge velocity

$\rho=$ Density of the fluid $^{[11,12]}$

$\mathrm{D}=$ Diameter of a pipe

$\mathrm{V}=$ Pore velocity $\left(\mathrm{m} \mathrm{sec}^{-1}\right)$

For porous media, the length scale corresponds to the hydraulic radius, $R_{H}$, while the velocity scale is the discharge velocity $\bar{u}$, divided by the porosity $\phi^{[1,3]}$.

Therefore, for porous medium, the Reynolds number can be rewritten as:

$$
\begin{aligned}
\operatorname{Re} & =\frac{4 R_{H} \bar{u} \rho}{\phi \mu} \\
& =\frac{2}{3(1-\phi)} \frac{D_{P} \bar{u} \rho}{\mu}
\end{aligned}
$$

Usually for convince of definition the numerical constants are dropped and in some definitions the (1- $\phi)$ term is also dropped. The Reynolds number then becomes:

$$
\operatorname{Re}_{\mathrm{P}}=\frac{\mathrm{D}_{\mathrm{P}} \overline{\mathrm{u}} \rho}{\mu}
$$

For the case of porous media used in this study which represents the DPF and the flow which is represented here by the exhaust gas emission having the following properties $\left(\overline{\mathrm{u}}=8 \mathrm{~m} \mathrm{sec}^{-1}, \rho_{\mathrm{g}}=0.35 \mathrm{~kg} \mathrm{~m}^{-3}\right.$, $\phi=0.45, \mu=4.15 \mathrm{E}^{-5}$ and $\left.\mathrm{D}=5 \mathrm{E}^{-6} \mathrm{~m}\right)$ and by applying Eq. 10 or 11 yields that: $\operatorname{Re}=0.408$, but at low temperatures the exhaust gases properties is changed ${ }^{[9,13]}$.

This results means that Darcy's law is applied for the case of flow in this type of porous media and that the flow is laminar.

\section{MATERIALS AND METHODS}

Porous media are considered now one of the famous and important media in sound and gas emission reduction, here these materials are studied and the flow properties through them is analyzed numerically.

Numerical solution for laminar flow in porous media: The flow in porous media is analyzed numerically using some appropriate software programming. This appropriate software program is used in order to find velocity distributions in both directions, $\mathrm{x}$ and $\mathrm{y}$ through the porous media and also to find temperatures profile and distribution through porous media. The results describe in addition the pressure distribution, wall shear and contours and profiles for temperature, pressure, velocities $V_{x}$ and $V_{y}$ for the flow in porous media which represents the a DPF unit made of silicon carbide $(\mathrm{SiC})^{[4,8]}$.

\section{RESULTS}

Figure 1 shows the relation between pressure drop along the typical DPF unit and the axial velocity and it can be noticed that as the velocity increases pressure drop also increase (as the velocity increase pressure decrease). Figure 2 shows pressure drop versus the axial velocity of gases flow through the different types of DPF unit, from figure it can be noticed that $\Delta \mathrm{P}$ for EX80: 200/14 DPF unit type is higher than other types, for RC: 200/14 it is the lowest. But at low velocities it can be noticed that EX80: 200/14 DPF unit type has the lowest pressure drop, hence it has a maximum values of transmission losses. Figure 3 shows the rate of change of VX, VY and pressure for the gas flow through the DPF unit at cold conditions, from this figure it can be noticed that all of these quantities decrease with the accumulative iteration number, i.e. with time and flow progress. This is a natural result since pressure and velocity of gasses emission are decreasing as the flow go through the filter, hence a pressure drop will occur and also the velocity of flow gases is decreased as the flow is in progress. Figure 4 shows the axial velocity variation profile for gasses emission through the porous media in the radial coordinate under cold conditions, the figure shows that there are fluctuations in the axial velocity variation with the continuity of gasses flow, because the porous DPF unit has variable nature i.e. sometimes rigid edges, pores and other variable

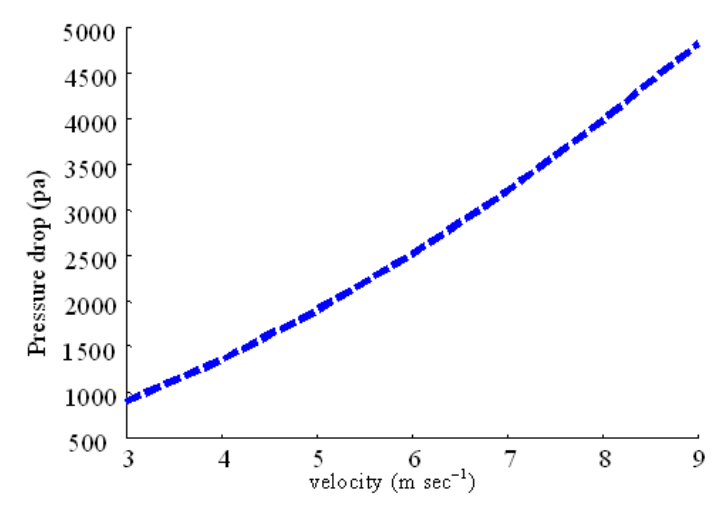

Fig. 1: Pressure drop against axial velocity for typical filter 


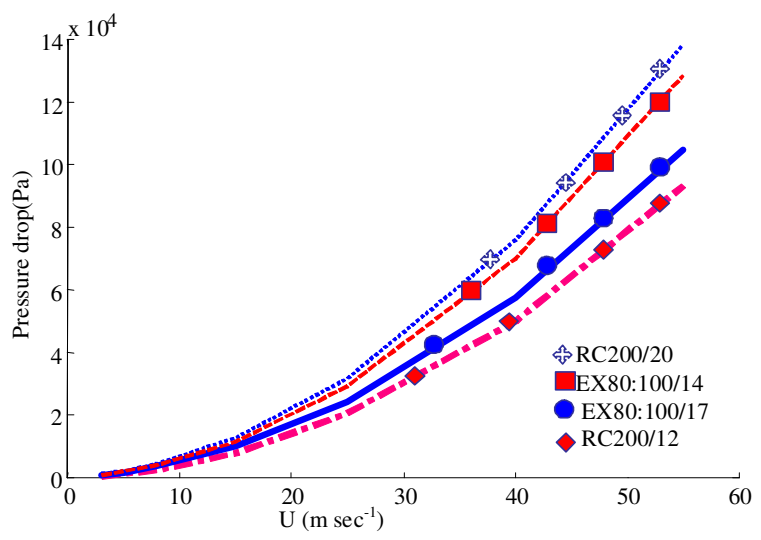

Fig. 2: Pressure drop for different types of DPF against velocity

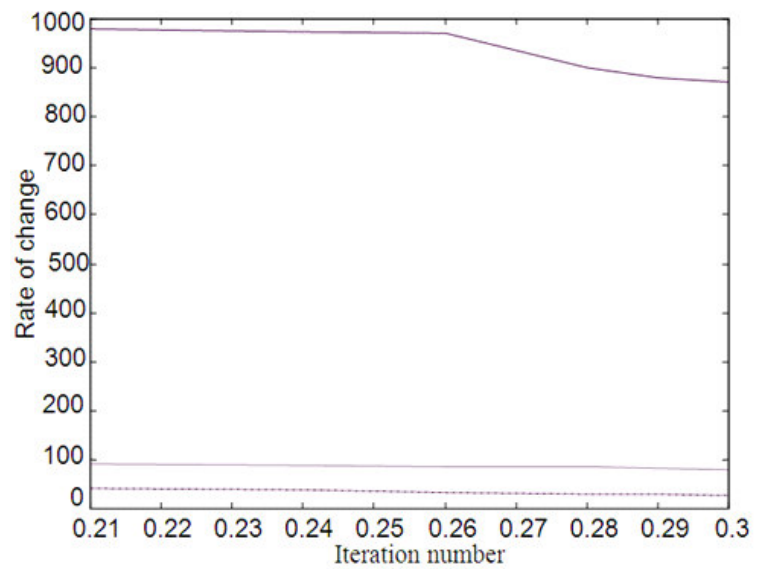

Fig. 3: VX (一) , VY*10(..-..-.-.) and Pressure(.....) rate of change for the gas flow through porous media under cold conditions

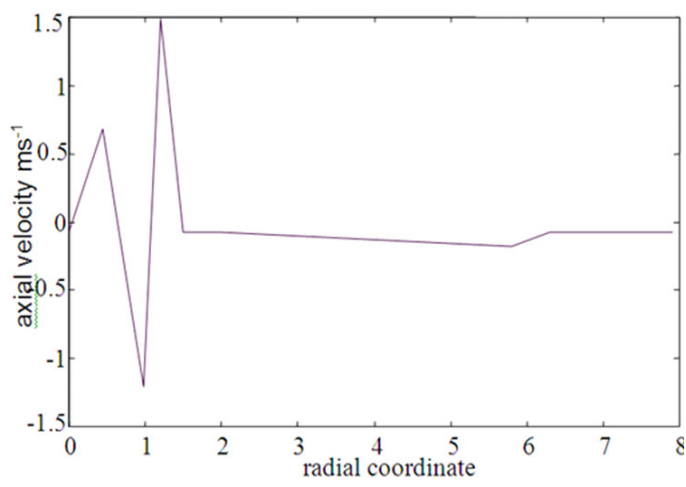

Fig. 4: Axial velocity profile for gases emission through the porous media under cold conditions

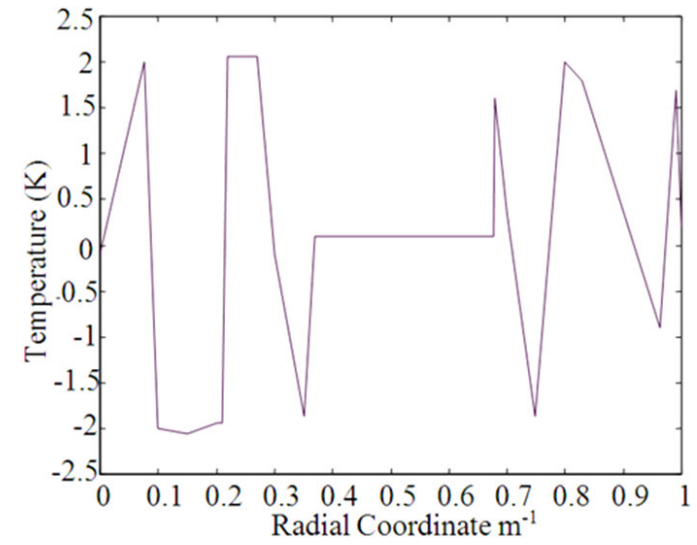

Fig. 5: Temperature variation profile for gases emission through the porous media under cold conditions

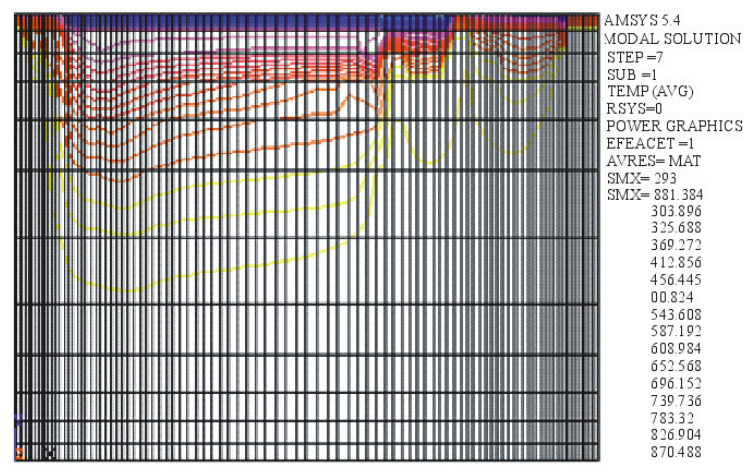

Fig.6: Temperature distributions through orous media under cold conditions

Figure 5 shows outlet temperature variation profile for gasses emission through the porous media in the radial coordinate under cold conditions, the figure shows that there are fluctuations in the outlet temperature with the continuity of gasses flow because of the nature of porous media.

Temperature distributions through the porous media under cold conditions are shown in Fig. 6. From last it is clear that the temperature decreases as the flow continued because the heat dissipated through the porous media and it will be either lose or dissipated by convection, radiation, or conduction. Figure 7 shows the rate of change of VX, VY and Pressure for the gas flow through the porous media under hot conditions and it can be noticed from figure that all these quantities suffer of depression with time of flow and this is a natural results because velocities, pressure and temperature decreases as the gasses flow through the porous media. 
Am. J. Engg. \& Applied Sci., 2 (1): 70-75, 2009

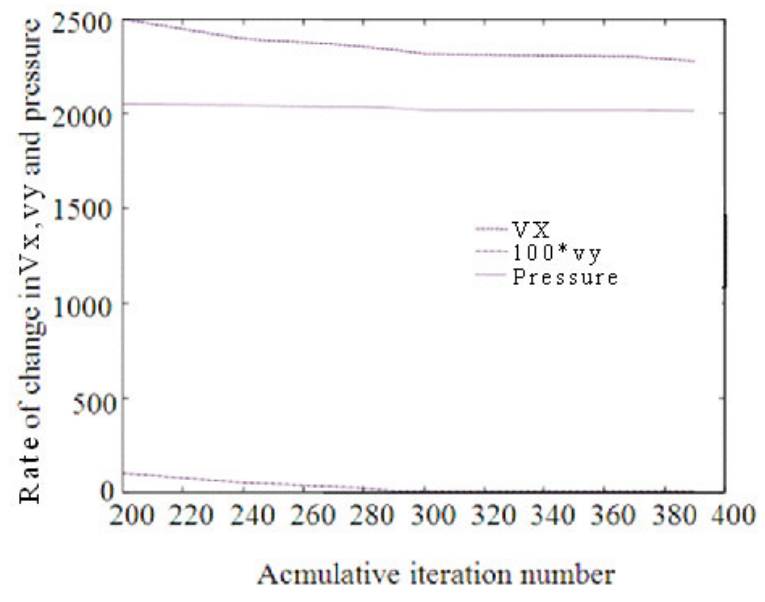

Fig. 7: VX, VY and Pressure rate of change for the gas flow through porous media under hot conditions

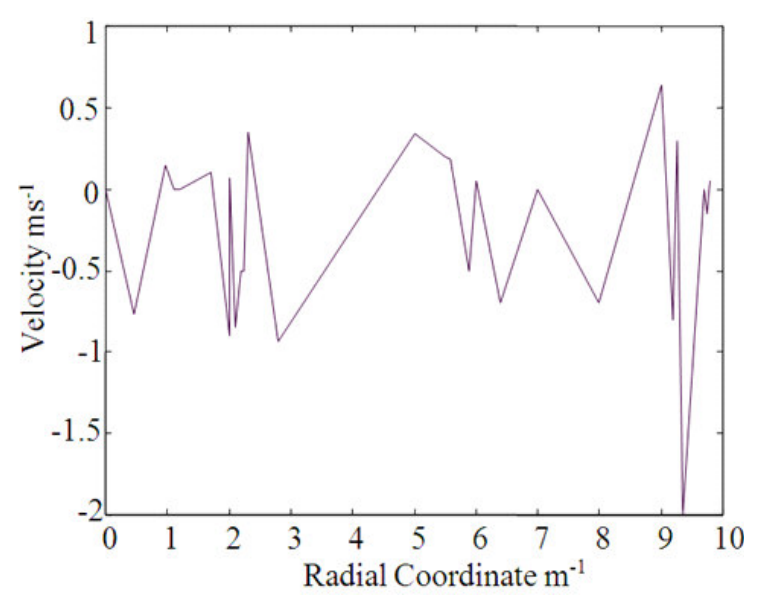

Fig. 8: Axial velocity profile for gases emission through the porous media under hot conditions

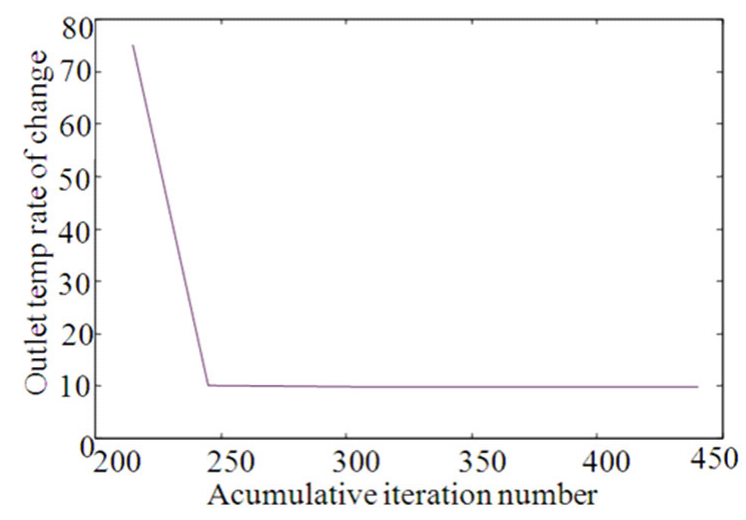

Fig. 9: Outlet temperature profile for gases emission through the porous media under hot conditions

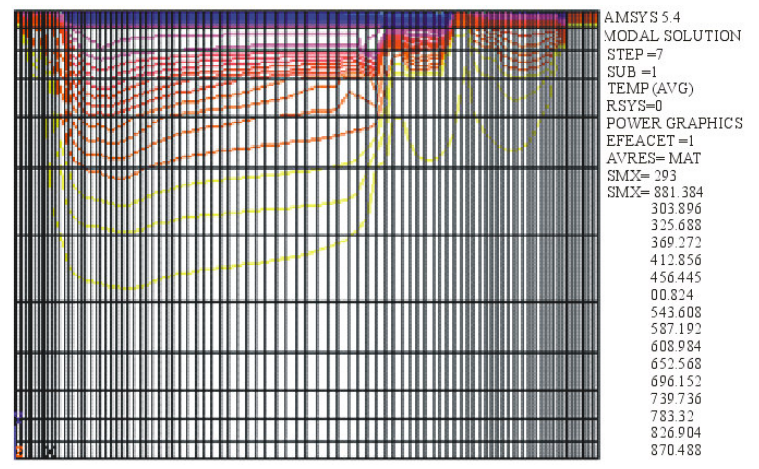

Fig. 10: Pressure distribution through the porous media under hot conditions
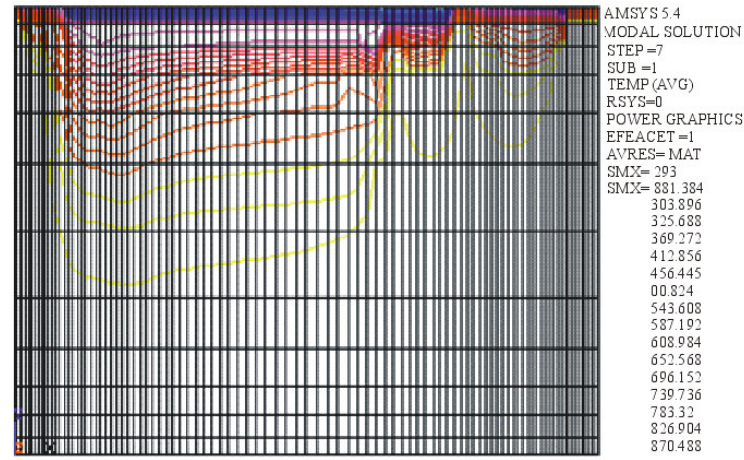

Fig. 11: Total pressure change across the porous media under hot conditions

Figure 8 shows the axial velocity variation profile for gasses emission through the DPF unit under hot conditions and big fluctuations can be noticed for axial velocity for the flow through the porous media, this fluctuation is resulted from the variation in axial velocity values as the gases in flow because of the change in nature of the porous media. Figure 9 shows the outlet temperature profile for exhaust gasses emission at hot conditions. From last two figures it can be noticed that both axial velocity and outlet temperature are decreases as the gasses flow is continued, but axial velocity fluctuation is more.

Figure 10 shows the pressure distribution through the porous media under hot conditions, from this figure it can be noticed that the pressure decreases down the pipe from inlet to outlet and this creates the pressure drop. Figure 11 shows the total pressure change across the porous media at hot conditions, from last figure it can be noticed that the total pressure is increased transversely because the sound pressure increases by some perturbation values. 


\section{DISCUSSION}

From last figures, it can be noticed that there is a pressure drop along the DPF unit as the gasses flow through this filter because it expands and so the area increase, then the pressure will decrease. Temperature values and distributions are fluctuating and vary from place to another along the exhaust and DPF unit, since as these gasses flow along the system there will be a heat losses, temperature absorbing materials ...etc. Both axial and vertical velocities are decreasing as the gases flow along the DPF unit because they loss some of kinetic energy and there may be some collisions and absorbing materials.

\section{CONCLUSION}

The main conclusions are:

- Gases pressure is decreased as such gases flow along the DPF unit and exhaust system, so there is a pressure drop.

- Temperature distribution and profiles show that the values of temperature have a fluctuation along the DPF unit.

- Axial and vertical velocities are decreasing as the gases flow through the DPF unit and the exhaust system.

\section{REFERENCES}

1. De Wiest, R.J.M., 1969. Flow through Porous Media. 3rd Edn., Academic Press, Inc. New York, USA-1969-12. pp: 5-45. ISBN-13:9780122142505.

2. Dokumaci, E., 1998. On transmission of sound in circular and rectangular narrow pipes with superimposed mean flow. J. Sound Vibration, 210: 375-389. DOI: 10.1006/JSVI.1997.1336 .

3. Holzbecher, E.O., 1998. Modeling Density-Driven Flow in Porous Media. 3rd Edn, 288P., Springerverlag Berlin, Germany, pp: 5-50. ISBN: 3-45063677.
4. Konstandopoulos, A.G. and J.H. Johnson, 1989. Wall-Flow Diesel Particulate Filters their Pressure Drop and Collection Efficiency. http://www.sae.org/technical/papers/890405

5. Klinkenberg, 1995 . Petroleum Reservoir Engineering. 1st Edn., USA., pp: 1-100.

6. Keith, A., 1982. Acoustical characteristics of porous materials. Phys. Rep., 82: 179-227. DOI: 10.1016/0370-1573(82)90131-4

7. Martys, N.S., 2001. Improved approximation of the brinkman equation using a lattice boltzman method. Phys. Fluids, 13: 1807-1810. DOI: 10.1063/1.1368846.

8. Muter, J., 1998. Predicting diesel particulate filters performance. Diesel Controls Limited, pp: 1-5.

9. Qh, S.K., D.S. Baik and Y.C. Han, 2002. Performance and exhaust gas characteristics on DPF trap. Int. J. Automotive Tech., 3: 111-115. http://society.kisti.re.kr/ Eksae/_notes/data/pdf/v3 n3_4.pdf

10. Silin, D., V. Korneev 2003. Pressure Diffusion Waves in Porous Media. Lawrence Berkeley National Laboratory, Gennady Goloshubin, University of Houston , paper LBNL, 52536-EXTAbs. $\quad$ pp: 1-4. http:/respositories.cdlib.org/lbnl/LBNL-54459.

11. Stanley, H., J. Andrade, M. Almeida, J. Mendes, S. Havlin and B. Suki, 1997. Fluid flow through porous media. Phys. Rev. Lett., 79: 21. DOI:10.1103/PpysRevLETT.79.3901.

12. Yu, R. and S.M. Shahed, 1981. Effects of Injection Timing and Exhaust Gas Recirculation on Emissions from a DI Diesel Engine. http://www.sae.org/technical/papers/811234.

13. Zheng, H. and J. Keith, 2004. Ignition analysis of wall-flow monolith diesel particulate filters. J. Catalysis Today, 98: 403-412. DOI: 10.1016/J. cattod.2004.08.008. 